Method A neonatal research nurse was recruited in 2012. Measures were implemented to increase the number and complexity of studies, and to be proactive in effective screening mechanisms, resulting in early identification of patients. Research was actively promoted, and teaching provided on a rolling programme. A close link was established with the Paediatric Research Nursing Team, to provide administrative support and cover in times of absence.

Links were made with research colleagues both regionally and nationally, and attendance at study days and conferences were identified as effective networking strategies.

Parental participation in research was encouraged, with the research nurse providing a link for the parents.

Results The number of studies has increased from an average of 1 ongoing study to 7 current studies. These studies include a meningitis study, a vaccine study, a platelet transfusion study and a large cohort observational study. A Patient Participation Involvement study is planned for the near future, and a service evaluation of the research team is awaiting approval.

Conclusions Effective neonatal research is dependent on a motivated and adequately resourced research team, including dedicated nursing research time. This has ensured that our LNNU will continue to provide excellent neonatal care, underpinned by research

\section{PO-0896 CONSEQUENCES OF SECOND - AND THIRDHAND SMOKE EXPOSURE FOR NEWBORNS AT THE NEONATAL INTENSIVE CARE UNIT}

KE de Jonge, MJ Hemmink, JM Wielenga. Intensive Care Neonatology, Emma Children's Hospital/Academic Medical Center, Amsterdam, Netherlands

\subsection{6/archdischild-2014-307384.1519}

Background and aims Smoking is consuming the smoke of smouldering tobacco. Inhaling harmful substances from tobacco and a higher risk for disorders, is general knowledge. Less well known are the possible effects of second- and thirdhand smoke. Secondhand smoking is the involuntary inhalation of tobacco smoke in the ambient air. Inhaling the smoke residues from walls, furniture, clothes, toys and other objects or absorbing it through the skin is referred to as thirdhand smoking. The aim is to provide a scientifically based advice on dealing with this issue in a hospital setting.

Methods A literature-study is performed. A PICO (patient-intervention-comparison-outcome) question is formulated to guide a literature search in scientific databases. Articles will be critically appraised.

Results Four studies (two reviews, one cohort, one descriptive) were found all pointing out that exposure to second- and thirdhand smoking may adversely affect young children, especially in the age up to 1 year including preterm or otherwise respiratory compromised newborn infants in the NICU. Also addressed is the lack of knowledge of parents and staff on the consequences of second-and thirdhand smoke. Hospital staff has an opportunity to educate parents about the effects of smoking on their newborn baby. Additional steps as changing clothes and wearing gloves after smoking would be appropriate.

Conclusions Healthcare professionals should take their responsibilities in preventing harm to fragile newborn infants as a result of tobacco smoke more serious.

\section{P0-0897 SUDDEN INFANT DEATH SYNDROME IN LOW BIRTH WEIGHT INFANTS}

V Cirik, E EFE. Nursing Faculty, Akdeniz University, Antalya, Turkey

\subsection{6/archdischild-2014-307384.1520}

Background Sudden infant death syndrome (SIDS) occurs less frequently in the first month of life, peaks between 2 and 4 months of age, and decreases thereafter. Prone sleeping (placing an infant to sleep on his/her stomach), bed sharing, parents not sleeping in the same room as the infant, an infant not using a pacifier during sleep, overheating, and maternal smoking during pregnancy have been suggested as contributing factors for SIDS.

Aim To examine SIDS in low birth weight infants.

Methods Compilation.

Results Infants who are born prematurely or who have low birthweight have up to four times the risk of SIDS than those infants born at term, and this risk increases with decreasing gestational age or birthweight. Low birth weight infants have a significantly higher risk of SIDS, a risk tightly correlated with prematurity. High SIDS incidence among low birth weight, very low birth weight, and extremely low birth weight infants persists despite increased overall survival within these infant populations. As a result, an increase in SIDS deaths could be expected in the lower birth weight infants because of the decreased competing mortality pressures during the neonatal period. In low birth weight and normal birth weight infants, we would anticipate a less marked increase in survival and, because of their increased maturity, a less apparent effect on SIDS rates.

Conclusion Parents of low birth weight infants require appropriate SIDS prevention education, including information about the dangers of environmental tobacco exposure and prone infant sleep.

\section{PO-0898 UMBILICAL CATHETER AND BLOOD SAMPLING IN VERY LOW BIRTH WEIGHT INFANTS FOR THE FIRST 72 HOUR OF LIFE PROTOCOL}

P Gil Castro, A Merino Landry, P Romero Pacheco, J Silla Gil. Neonatology, Hospital Sant Joan de Déu, Barcelona, Spain

\subsection{6/archdischild-2014-307384.1521}

Background and aims Very low birth weight (VLBW) infants are extremely vulnerable and usually require central catheters and frequent blood tests. Our aim was to develop a protocol regarding umbilical catheter fixation and blood samples for VLBW during the first $72 \mathrm{~h}$ of life.

Methods A retrospective study was carried out by a multidisciplinary team describing the intervention variables in 10 VLBW admitted to our Unit. In parallel a bibliographic research was performed on the International data bases, answering our PICO questions. The population of the study was VLBW under 32 weeks of life and/or under 1.500 grams of weight. Interventions were catheter fixation, number, volume and speed of extraction and reinfusion during first $72 \mathrm{~h}$.

Results Our population had a mean gestational age and birth weight of 30 weeks and 1200 grams. Our data showed a mean of 11 tests per patient during first $72 \mathrm{~h}$ of life, none using umbilical cord blood. Registration of velocity was not reported. After a literature review we designed a protocol of catheter fixation and blood samples for VLBW during first $72 \mathrm{~h}$. The protocol included using cord blood for the first sampling and a description of adequate sample size and velocities. 
Conclusions An evidence based practice leads to improve blood sampling in VLBW infants. Post intervention results are still to be measured.

\section{PO-0899 THE IMPLEMENTATION RATE OF DEVELOPMENTAL CARE IN IRANIAN NICUS}

'ZZ Godarzi, ${ }^{2} \mathrm{O}$ Rahimi, ${ }^{3} \mathrm{~N}$ Khalesi, ${ }^{4} \mathrm{~A}$ Shamshiri, ${ }^{5} \mathrm{~N}$ Mohammadi. ${ }^{1}$ Neonatal Intensive Group, Faculty Nursing and Midwifery of Tehran University of Medical Sciences, Tehran, Iran; ${ }^{2}$ Neonatal Intensive Care Unit, Bahrami Hospital Tehran University of Medical Sciences Tehran Iran (E-Mail, Tehran, Iran; ${ }^{3}$ Medicin Teaching, Associate Professor School of Medicine Tehran University of Medical Sciences., Tehran, Iran; ${ }^{4}$ Epidemiologist Unit, Dentistry Research Institute and Department of Community Oral Health School of Dentistry Tehran University of Medical Sciences, Tehran, Iran; ${ }^{5}$ Critical Care Group, Faculty Nursing and Midwifery Tehran University of Medical Sciences, Tehran, Iran

\subsection{6/archdischild-2014-307384.1522}

Background Nowadays, Developmental Centred Care in NeonatalIntensive Care Units (NICU) is expanding and being implemented in developedcountries in the form of scientific competition. The aim of this study was to evaluate the implementation rate of developmental centeredcare in NICUs in Tehran University of Medical Sciences hospitals in Tehran, Iran.

Methods A descriptive cross-sectional study was conducted in ninehospitals NICUs in 2012. The sample was included 138 nurses who had working intheir professional work. Using a selfadministrated questionnaire on coremeasure for checking the implementation of developmental care in NICUs anddemography characteristic.

Results The finding which indicated that, the highest rate ofdevelopmental centred care implementation, with $79.46 \%$ was routine care. Followed by sleep care $65.43 \%$, providing ahealthy environment $65.27 \%$ and family-centred care $63.32 \%$, respectively. Meanwhile,the lowest rate was $59.16 \%$ for the implementation of pain, stress managementand assessment.

Conclusion This study result which indicates that the implementationrate of the developmental centred care aspects in NICUs is low. Therefore,immediate actions should be taken for further promotion of developmental care,development of professional training and more importantly development ofinstruction and further research is needed.

\section{PO-0900 TRANSLATION COMFORTNEO - A PAIN ASSESSMENT TOOL}

R Stenkjaer, M Andersen, M Scheutz, Y Hundrup. Neonatalklinikken, Rigshospitalet University of Copenhagen, Copenhagen, Denmark

\subsection{6/archdischild-2014-307384.1523}

Background COMFORTneo scale is a pain assessment tool designed to assess the degree of pain for neonates. The scale is designed to assess the short term or long term pain. Centre for Clinical guidelines recommend the use of COMFORTneo systematic assessment of pain in newborns in Denmark. The instrument was originally developed in the Netherlands, but are published in an English edition.

Purpose The purpose of this poster is to describe how the pain assessment tool COMFORTneo has been translated into Danish and adapted to Danish conditions.

Method We have followed the principles recognised as good practice for the translation and cultural adaptation of an instrument from the source language into another language. This translation process involves eight steps: 1) Preparation 2) Forward translation 3) Comparison and critical review of the translations 4) Back Translation 5) Review of back translation 6) Adjustment of COMFORTneo to Danish conditions 7) Pilot Testing and 8) Proof and final adoption.

Results Translation and adaptation of COMFORTneo has been a fruitful process. Immediately, the applicability of COMFORTneo was confirmed through involvement of many nurses in the translation process. Involvement of nurses has been important and theese comments have contributed to the establishment of a Danish version of COMFORTneo that makes sense for Danish nurses.

Perspectives Following good practice for the translation and cultural adaptation, an instrument COMFORTneo is ready for implementation. This is of great importance as instruments of pain scoring of premature and sick newborns and are called for by many Danish neonatal departments.

\section{P0-0901 NEONATAL OUTCOMES OF ADOLESCENT PREGNANCIES IN A SECONDARY LEVEL MATERNITY HOSPITAL IN ROMANIA}

${ }^{1} \mathrm{LM}$ Suciu, ${ }^{1} \mathrm{M}$ Simon, ${ }^{2} \mathrm{R}$ Peti, ${ }^{2} \mathrm{M}$ Luca, ${ }^{1} \mathrm{M}$ Cucerea. ${ }^{1}$ Pediatrics, University of Medicine and Pharmacy Tirgu Mures, Tirgu Mures, Romania; ${ }^{2}$ Neonatology, County Hospital Tirgu Mures, Tirgu Mures, Romania

\subsection{6/archdischild-2014-307384.1524}

Background and aims Pregnant adolescents have high rates of poor birth outcomes, but the causes are unclear. There is a very marked association between young age of mothers and low birth weight and preterm delivery and some of the apparent effect of young maternal age on birth weight may be because the birth is likely to be the mother's first, and first births have a higher incidence of prematurity. The aim of present study was to investigate neonatal outcomes of teenage pregnancies controlling for parity, gestational age and perinatal interventions.

Methods A retrospective study comparing singleton deliveries classified into three teenage groups:12-15, 15-17, 18-19, and a comparison group of 24-29 years was performed in a secondary level Maternity Hospital in Mures County, Romania. For the adult group, 736 charts of mothers between the age of 24 and 29 delivering singleton babies were selected.

Results The study population consisted of 1131 women, 75 12-15 years old, 163 16-17 years old, 157 18-19 years old, and 736 24-29 years old. A significant linear association was found between maternal age and preterm birth, low birth weight, and neonatal birth related trauma. Length of hospitalisation, as a marker of the healthcare costs involved in the care of these high risk cases, was significant associated with maternal age after controlling for perinatal interventions.

Conclusions Teenage pregnancy is a risk factor for low birth weight, preterm delivery, neonatal birth related trauma and high healthcare costs.

\section{PO-0902 ANNP (ADVANCED NEONATAL NURSE PRACTITIONER) WORKFORCE: BALANCING SERVICE PROVISION AND TRAINING IN A LOCAL NEONATAL UNIT (NNU)}

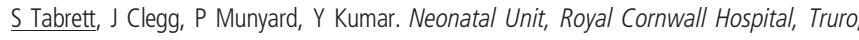
UK

10.1136/archdischild-2014-307384.1525 